\title{
Complex Transmission Eigenvalues in One Dimension
}

\author{
Yalin Zhang, ${ }^{1}$ Yanling Wang, ${ }^{1}$ Guoliang Shi, ${ }^{1}$ and Shizhong Liao ${ }^{2}$ \\ ${ }^{1}$ Department of Mathematics, Tianjin University, Tianjin 300072, China \\ ${ }^{2}$ School of Computer Science and Technology, Tianjin University, Tianjin 300072, China
}

Correspondence should be addressed to Yanling Wang; mathtju@163.com

Received 15 May 2014; Accepted 18 June 2014; Published 6 July 2014

Academic Editor: Ali H. Bhrawy

Copyright (c) 2014 Yalin Zhang et al. This is an open access article distributed under the Creative Commons Attribution License, which permits unrestricted use, distribution, and reproduction in any medium, provided the original work is properly cited.

We consider all of the transmission eigenvalues for one-dimensional media. We give some conditions under which complex eigenvalues exist. In the case when the index of refraction is constant, it is shown that all the transmission eigenvalues are real if and only if the index of refraction is an odd number or reciprocal of an odd number.

\section{Introduction}

The transmission eigenvalue problem appears in the inverse scattering theory for acoustic and electromagnetic waves [1]. It is a nonlinear boundary value problem for a coupled set of equations defined on the support of the scattering object. Since this eigenvalue problem is not self-adjoint, there exists the possibility of complex eigenvalues which has been proved for the spherically stratified media under some conditions in [2-4]. But so far only a small part of the transmission eigenvalues (real eigenvalues or complex eigenvalues) has been considered; this is of great limitation in the inverse scattering problem. If we consider all of the transmission eigenvalues, this problem, even for the one-dimensional media, is not simple. For one-dimensional problem, Sylvester [5] has shown how to locate all the transmission eigenvalues in the complex plane for a constant index of refraction. In this work we contribute more to the discussion of the onedimensional case:

$$
\begin{array}{cc}
u^{\prime \prime}+k^{2} n(x) u=0, & x \in[0,1], \\
v^{\prime \prime}+k^{2} v=0, & x \in[0,1], \\
u(0)=v(0), & u(1)=v(1), \\
u^{\prime}(0)=v^{\prime}(0), & u^{\prime}(1)=v^{\prime}(1) .
\end{array}
$$

We refer to the set of all $k \in \mathbb{C}$ for which (1) has nontrivial solutions as the transmission eigenvalues. The corresponding nontrivial solutions $(u, v)$ are called the transmission eigenfunctions. Throughout this paper, we assume that

$$
\begin{gathered}
n(x)>0, \quad x \in[0,1] ; \\
n \in C^{1}(0,1) ; \quad n^{\prime \prime} \in L^{2}(0,1) ; \quad n^{\prime}(0)=0 .
\end{gathered}
$$

Since only real eigenvalues can be determined from the scattering data and the physical properties of the scattering object can be obtained from the transmission eigenvalues, it is of interest to find the existence conditions of the complex transmission eigenvalues and to research the conditions under which there are no complex eigenvalues at all. For the existence of the transmission eigenvalues, to the author's knowledge, only the sufficient conditions are given.

The plan of our paper is as follows. In Section 2, motivated by [3] which gives the existence conditions of complex transmission eigenvalues for three-dimensional media, we turn our attention to the case when $n(r)$ is a variable and show that complex eigenvalues can exist. Then, in Section 3, using the methods in $[3,5]$, we give the necessary and sufficient condition for the existence of complex transmission eigenvalues when the index of refraction is a constant.

\section{The Existence of Complex Transmission Eigenvalues for Variable $n(x)$}

Our research methods rely on transforming the first Helmholtz equation in (1) into a Sturm-Liouville form that separates $k$ and $n(x)$. 
Suppose that the fundamental solutions $u_{1}$ and $u_{2}$ satisfy the initial value problems:

$$
\begin{gathered}
u_{i}^{\prime \prime}+k^{2} n(x) u_{i}=0, \quad i=1,2, \\
u_{1}(0)=u_{2}^{\prime}(0)=0, \\
u_{1}^{\prime}(0)=u_{2}(0)=1 .
\end{gathered}
$$

Then the solutions $u$ and $v$ which satisfy (1) can be written as

$$
\begin{aligned}
& u(x)=a_{1} u_{1}(x)+a_{2} u_{2}(x), \\
& v(x)=b_{1} \sin k x+b_{2} \cos k x,
\end{aligned}
$$

for constants $a_{1}, a_{2}, b_{1}$, and $b_{2}$.

Lemma 1. The value $k$ is a transmission eigenvalue if and only if $d(k)=0$, where

$$
\begin{aligned}
d(k)= & -2+u_{1}(1) k \sin k-u_{2}^{\prime}(1) \frac{\sin k}{k} \\
& +u_{1}^{\prime}(1) \cos k+u_{2}(1) \cos k,
\end{aligned}
$$

where $u_{1}$ and $u_{2}$ are the solutions of (3).

Proof. The boundary conditions in (1) imply that

$$
\begin{gathered}
a_{2}=b_{2}, \\
a_{1}=b_{1} k, \\
a_{1} u_{1}(1)+a_{2} u_{2}(1)=b_{1} \sin k+b_{2} \cos k, \\
a_{1} u_{1}^{\prime}(1)+a_{2} u_{2}^{\prime}(1)=b_{1} k \cos k-b_{2} k \sin k .
\end{gathered}
$$

So in order for the value $k$ to be a transmission eigenvalue there must exist a nontrivial pair $\left(a_{1}, a_{2}\right)$ satisfying

$$
\left(\begin{array}{cc}
u_{1}(1)-\frac{1}{k} \sin k & u_{2}(1)-\cos k \\
u_{1}^{\prime}(1)-\cos k & u_{2}^{\prime}(1)+k \sin k
\end{array}\right)\left(\begin{array}{l}
a_{1} \\
a_{2}
\end{array}\right)=\left(\begin{array}{l}
0 \\
0
\end{array}\right) .
$$

For this to be true the determinant of the coefficient matrix must be zero:

$$
d(k):=\operatorname{det}\left(\begin{array}{cc}
u_{1}(1)-\frac{1}{k} \sin k & u_{2}(1)-\cos k \\
u_{1}^{\prime}(1)-\cos k & u_{2}^{\prime}(1)+k \sin k
\end{array}\right)=0 .
$$

Using the Wronskian identity for the fundamental solutions of the Sturm-Liouville problems

$$
\begin{aligned}
W\left(u_{1}, u_{2}\right)(x) & :=u_{1}(x) u_{2}^{\prime}(x)-u_{1}^{\prime}(x) u_{2}(x) \\
& =-1 \text { in }[0,1],
\end{aligned}
$$

we have

$$
\begin{aligned}
d(k)= & -2+u_{1}(1) k \sin k-u_{2}^{\prime}(1) \frac{\sin k}{k} \\
& +u_{1}^{\prime}(1) \cos k+u_{2}(1) \cos k .
\end{aligned}
$$

The proof is now complete.
The determinant condition $d(k)=0$ gives us an algebraic relation that must be satisfied by the transmission eigenvalues. This reduces the study of the transmission eigenvalue problem to a root finding problem. Next, we will give the expansions of $u_{1}, u_{1}^{\prime}$ and $u_{2}, u_{2}^{\prime}$. We make the change of variables

$$
t=\int_{0}^{x} \sqrt{n(s)} d s
$$

$$
y_{i}(t)=(n(x))^{1 / 4} u_{i}(x), \quad i=1,2 .
$$

Since $n$ satisfies (2), the problem (3) becomes

$$
\begin{gathered}
y_{i}^{\prime \prime}(t)+\left(k^{2}-p(t)\right) y_{i}(t)=0 \quad \text { for } t \in[0, \delta] ; \\
y_{1}(0)=0, \quad y_{1}^{\prime}(0)=n(0)^{-1 / 4}, \\
y_{2}(0)=n(0)^{1 / 4}, \quad y_{2}^{\prime}(0)=0,
\end{gathered}
$$

where

$$
\begin{gathered}
p(t)=\frac{1}{4} \frac{n^{\prime \prime}(x)}{n(x)^{2}}-\frac{5}{16} \frac{n^{\prime}(x)^{2}}{n(x)^{3}}, \\
\delta=\int_{0}^{1} \sqrt{n(s)} d s .
\end{gathered}
$$

With the help of the Liouville transformation (11) and some basic estimates in [6] for the corresponding Schrödinger equations in (12), we get the following lemma.

Lemma 2. Assume that $n$ satisfies (2). Then there exists a positive constant $C$ such that, for all $x \in[0,1]$ and $k \in \mathbb{C}$, the solutions $u_{1}(x), u_{2}(x)$ to (3), and their $x$-derivatives satisfy

$$
\begin{aligned}
& \left|u_{1}(x)-\frac{1}{[n(0) n(x)]^{1 / 4} k} \sin (k t(x))\right| \\
& \leq \frac{C}{\left|k^{2}\right|} \exp (|\operatorname{Im} k| t(x)), \\
& \left|u_{1}^{\prime}(x)-\left(\frac{n(x)}{n(0)}\right)^{1 / 4} \cos (k t(x))\right| \\
& \leq \frac{C}{|k|} \exp (|\operatorname{Im} k| t(x)), \\
& \left|u_{2}(x)-\left(\frac{n(0)}{n(x)}\right)^{1 / 4} \cos (k t(x))\right| \\
& \leq \frac{C}{|k|} \exp (|\operatorname{Im} k| t(x)), \\
& \left|u_{2}^{\prime}(x)+k(n(0) n(x))^{1 / 4} \sin (k t(x))\right| \\
& \leq C \exp (|\operatorname{Im} k| t(x)),
\end{aligned}
$$

where $t(x)=\int_{0}^{x} \sqrt{n(s)} d s$.

So we have the following asymptotic expansions. 
Lemma 3. Assume that $n$ satisfies (2). Then for all $x \in[0,1]$, as $|k| \rightarrow \infty$ in $\mathbb{C}, u_{1}(x), u_{2}(x)$, and their $x$-derivatives satisfy

$$
\begin{aligned}
u_{1}(x)= & \frac{1}{[n(0) n(x)]^{1 / 4} k} \sin (k t(x)) \\
& +O\left(\frac{\exp (|\operatorname{Im} k| t(x))}{k^{2}}\right), \\
u_{1}^{\prime}(x)= & \left(\frac{n(x)}{n(0)}\right)^{1 / 4} \cos (k t(x)) \\
& +O\left(\frac{\exp (|\operatorname{Im} k| t(x))}{k}\right), \\
u_{2}(x)= & \left(\frac{n(0)}{n(x)}\right)^{1 / 4} \cos (k t(x)) \\
& +O\left(\frac{\exp (|\operatorname{Im} k| t(x))}{k}\right), \\
& +O(\exp (|\operatorname{Im} k| t(x))), \\
u_{2}^{\prime}(x)= & k(n(0) n(x))^{1 / 4} \sin (k t(x))
\end{aligned}
$$

where $t(x)=\int_{0}^{x} \sqrt{n(s)} d s$.

After substituting the asymptotic expansions of $u_{1}$ and $u_{2}$ into (5), we have

$$
\begin{aligned}
d(k)= & -2+B \sin (k \delta) \sin k+C \cos (k \delta) \cos k \\
& +O\left(\frac{\exp (|\operatorname{Im} k|(\delta+1))}{k}\right),
\end{aligned}
$$

as $|k| \rightarrow \infty$, where

$$
\begin{gathered}
B=\frac{1}{(n(0) n(1))^{1 / 4}}+(n(0) n(1))^{1 / 4}, \\
C=\left(\frac{n(1)}{n(0)}\right)^{1 / 4}+\left(\frac{n(0)}{n(1)}\right)^{1 / 4}, \\
\delta=\int_{0}^{1} \sqrt{n(s)} d s .
\end{gathered}
$$

According to the fundamental inequality $a^{2}+b^{2} \geq 2 a b$ for $a, b>0$, we know $B \geq 2$ and $C \geq 2$. If $n(0)=1$ or $n(1)=1$, then $B=C$ and the term $-2+B \sin (k \delta) \sin k+C \cos (k \delta) \cos k$ in (16) becomes $-2+C \cos (k(\delta-1))$ which is a periodic function if $\delta$ is rational and almost periodic if $\delta$ is irrational (see [7]). The fact that $C \geq 2$ means that, for large enough $k, d(k)$ has infinitely many real zeros if we assume $\delta \neq 1$. Otherwise, if $\delta=1$, we have that

$$
\begin{aligned}
d(k) & -O\left(\frac{\exp (|\operatorname{Im} k|(\delta+1))}{k}\right) \\
& =-2+B \sin ^{2} k+C \cos ^{2} k \\
& =-2+\frac{B+C}{2}+\frac{C-B}{2} \cos (2 k) .
\end{aligned}
$$

So if only one of the values $n(0)$ and $n(1)$ is 1 , then $B=C>2$, and the values $k \in \mathbb{R}$ are not transmission eigenvalues in the case when $|k| \rightarrow \infty$.

Our aim here is to find conditions under which $d(k)$ has an infinite number of complex zeros when $n(0) \neq 1, n(1) \neq 1$. The search for zeros of $d(k)$ leads us to look for the zeros of the polynomial

$$
T(k):=-2+B \sin (k \delta) \sin k+C \cos (k \delta) \cos k .
$$

We assume that $\delta$ is a rational number, and $\delta=m / n>1$, $m, n \in \mathbb{Z}^{+}$. Replacing $k$ with $n k$, we get

$$
T(n k)=-2+B \sin (m k) \sin (n k)+C \cos (m k) \cos (n k) \text {. }
$$

It is obviously true that $\left|e^{i k}\right|=1$ if $k \in \mathbb{R}$; that is, $\left|e^{i k}\right| \neq 1$ implies that $k \notin \mathbb{R}$. Based on this fact, a substitution of $z=e^{i k}$ into (20) is used. According to Euler's formula, we have

$$
\begin{aligned}
& \sin (m k)=\frac{e^{i m k}-e^{-i m k}}{2 i}=\frac{z^{m}-z^{-m}}{2 i}, \\
& \cos (m k)=\frac{e^{i m k}+e^{-i m k}}{2}=\frac{z^{m}+z^{-m}}{2} .
\end{aligned}
$$

So (20) becomes

$$
-2-\frac{B}{4}\left(z^{m}-z^{-m}\right)\left(z^{n}-z^{-n}\right)+\frac{C}{4}\left(z^{m}+z^{-m}\right)\left(z^{n}+z^{-n}\right) .
$$

We assume that $n(0) \neq 1$ and $n(1) \neq 1$; that is, $B \neq C$. Multiplying $(22)$ by $(4 /(B-C)) z^{m+n}$ leads us to look for zeros of the polynomial

$$
\begin{aligned}
& p(z) \\
& :=\frac{-8 z^{m+n}-B\left(z^{2 m}-1\right)\left(z^{2 n}-1\right)+C\left(z^{2 m}+1\right)\left(z^{2 n}+1\right)}{B-C} \\
& =-z^{2(m+n)}+\frac{B+C}{B-C} z^{2 m}-\frac{8}{B-C} z^{m+n}+\frac{B+C}{B-C} z^{2 n}-1 .
\end{aligned}
$$

In order to show the conditions under which $T(k)$ has complex zeros, we only need to research in what situations $p(z)$ cannot have all roots lying on the unit circle $|z|=1$. The above polynomial $p(z)$ is a self-inversive polynomial because

$$
z^{2(m+n)} p\left(\frac{1}{z}\right)=p(z) .
$$

Note that the zeros of a self-inversive polynomial either lie on $|z|=1$ or are symmetric with respect to the unit circle. We further have the following lemma (see [3]).

Lemma 4 (Cohn). Let $p(z)$ be a self-inversive polynomial. Then all the zeros of $p(z)$ lie on the unit circle if and only if all the zeros of $p^{\prime}(z)$ lie in $|z| \leq 1$.

Then we have the following result. 
Theorem 5. Assume that $n$ satisfies (2), $n(0) \neq 1, n(1) \neq 1$, and $\delta=\int_{0}^{1} \sqrt{n(s)} d s$ is a rational number greater than 1 . Then if either (28) or (33) is valid, the eigenvalue problem (1) has an infinite number of complex eigenvalues and all these eigenvalues lie in a strip parallel to the real axis.

Proof. Based on Cohn's theorem, our first aim is to look for conditions of $n$ under which there are some zeros of $p^{\prime}(z)$ lying outside $|z|=1$. For $p(z)$ that was defined in (23), we have

$$
\begin{aligned}
z p^{\prime}(z)= & -2(m+n) z^{2(m+n)}+2 m \frac{B+C}{B-C} z^{2 m}-\frac{8(m+n)}{B-C} z^{m+n} \\
& +2 n \frac{B+C}{B-C} z^{2 n} .
\end{aligned}
$$

With the help of Vieta theorem, the product of all zeros of (25) equals

$$
-\frac{n}{m+n} \frac{B+C}{B-C}
$$

If the absolute value of this product is greater than 1 , there is at least one zero lying outside the unite circle $|z|=1$. Hence we give the first condition

$$
\left|\frac{n}{m+n} \frac{B+C}{B-C}\right|>1
$$

In other words,

$$
\left|\frac{B+C}{B-C}\right|>1+\delta
$$

By Rouchés theorem, we can derive another condition for (25) to have zeros outside the unite circle $|z|=1$. Set

$$
\begin{aligned}
q(z):= & \frac{z^{2(m+n)}}{2} \frac{1}{z} p^{\prime}\left(\frac{1}{z}\right) \\
= & -(m+n)+m \frac{B+C}{B-C} z^{2 n} \\
& -\frac{4(m+n)}{B-C} z^{m+n}+n \frac{B+C}{B-C} z^{2 m} .
\end{aligned}
$$

Then we need to prove that $q(z)$ has zeros in $|z|<1$. On the unite circle $|z|=1$,

$$
\begin{aligned}
& \left|q(z)-m \frac{B+C}{B-C} z^{2 n}\right| \\
& =\left|-(m+n)-\frac{4(m+n)}{B-C} z^{m+n}+n \frac{B+C}{B-C} z^{2 m}\right| \\
& \leq m+n+\frac{4(m+n)}{|B-C|}+n \frac{B+C}{|B-C|}, \\
& \quad\left|m \frac{B+C}{B-C} z^{2 n}\right|=m \frac{B+C}{|B-C|} .
\end{aligned}
$$

Therefore

$$
\left|q(z)-m \frac{B+C}{B-C} z^{2 n}\right|<\left|m \frac{B+C}{B-C} z^{2 n}\right|,
$$

if

$$
m+n+\frac{4(m+n)}{|B-C|}+n \frac{B+C}{|B-C|}<m \frac{B+C}{|B-C|} .
$$

Rouché's theorem implies that $q(z)$ has $2 n$ zeros inside the unit disc. So $p^{\prime}(z)$ has $2 n$ zeros outside the unit disc from (29). Condition (32) can be stated as

$$
\frac{|B-C|+4}{B+C}<\frac{\delta-1}{1+\delta}
$$

So far, we obtain two conditions (28) and (33) which guarantee the existence of complex zeros of $T(k)$.

The following proof for the existence of complex zeros for $d(k)$ is the same as that stated in [3]. To facilitate reading, we state it once again. If $p(z)$ has zeros not on the unite circle $|z|=1(T(k)$ has complex zeros in this case), then $p(z)$ has zeros outside the unit circle, and the zeros inside the unite circle $|z|=1$ have a positive distance from the origin. Suppose those zeros are $z_{j}=r_{j} e^{i \theta_{j}}, j=1, \ldots, h, h \leq m+n$, where $m$ and $n$ are two integers which are used to denote $\delta$. Based on the substitution $z=e^{i k}$, we know that each $z_{j}=r_{j} e^{i \theta_{j}}$ corresponds to the complex zeros $k_{j}=\theta_{j}+2 l \pi-i \log r_{j}$, $l=1,2, \ldots$, for $T(n k)$. Then the corresponding zeros of $T(k)$ are $n \theta_{j}+2 n l \pi-i n \log r_{j}$, where $l=1,2, \ldots$, and $n$ is a positive integer. So all these complex transmission eigenvalues stay inside the strip

$$
|\operatorname{Im} k| \leq n \max \left|\log r_{j}\right|,
$$

since $0<r_{j}<1$. Let $C_{j}$ be a small circle surrounding $z_{j}$, lie inside the unit circle, and isolate $z_{j}$ from the other zeros of $p(z)$. Under the transformation $z=e^{i k}$, the circle $C_{j}$ corresponds to a periodic array of closed Jordan curves surrounding each of the corresponding zeros of $T(k)$, and, on these curves, $|T(k)|>d_{j}$ for some constant $d_{j}>0$. From (16), we have that

$$
d(k)=T(k)+O\left(\frac{\exp (|\operatorname{Im} k|(\delta+1))}{k}\right)
$$

for $k$ being large enough. Using (34), we get that

$$
|d(k)-T(k)|<|T(k)|,
$$

is valid for $k$ being large and lying on some closed Jordan curves. It follows from Rouchés theorem that $d(k)$ has a complex zero inside each Jordan curve when $k$ is large.

Next, inspired by the results and methods in [4], we will show that, when $n(0) \neq 1, n(1) \neq 1$, if transmission eigenvalues exist they must lie in a strip parallel to the real axis; that is, we remove some assumptions on $n(x)$ which were required in the above theorem. The major tool we use is the following result from [8] for an entire function.

Lemma 6 (Paley-Wiener). Let $f(z)$ be an entire function such that

$$
|f(z)| \leq C e^{A|z|}
$$


for positive constants $A$ and $C$ and all values of $z$, and

$$
\int_{-\infty}^{\infty}|f(z)|^{2} d x<\infty
$$

Then there exists a function $\phi$ in $L^{2}[-A, A]$ such that

$$
f(z)=\int_{-A}^{A} \phi(t) e^{i z t} d t .
$$

Theorem 7. Assume that $n$ satisfies (2) and $n(0) \neq 1, n(1) \neq 1$. Then if complex eigenvalues exist, all of them lie in a strip parallel to the real axis.

Proof. From the identities $\cos (a-b)=\cos a \cos b+\sin a \sin b$ and $\cos (a+b)=\cos a \cos b-\sin a \sin b$, (19) implies that

$$
T(k)=-2+\frac{B+C}{2} \cos (k(\delta-1))-\frac{B-C}{2} \cos (k(\delta+1)) \text {. }
$$

Hence if $n(0) \neq 1, n(1) \neq 1$, then $B \neq C$. In this case, $T(k)$ is an entire function of $k$ of exponential type $\delta+1$. It follows from Lemma 2 that $u_{1}(1), u_{1}^{\prime}(1), u_{2}(1)$, and $u_{2}^{\prime}(1)$ are entire functions of $k$ of exponential type $\delta$. From (5), we get that $d(k)$ is an entire functions of $k$ of exponential type at most $\delta+1$. Then (35) implies that $E(k):=O((\exp (|\operatorname{Im} k|(\delta+1))) / k)$ is also an entire function of $k$ of exponential type at most $\delta+1$.

Furthermore, at $k=0, d(k)=0,|T(k)|=|-2+C|<\infty$; then $E(k)$ is an $L^{2}$ function on the real axis. So $E(k)$ meets the conditions in the Paley-Wiener theorem. Then there exists a function $\phi \in L^{2}[-(\delta+1),(\delta+1)]$ such that

$$
E(k)=\int_{-(\delta+1)}^{\delta+1} \phi(t) e^{i k t} d t
$$

Using Schwarz inequality, we have that

$$
\begin{aligned}
|E(k)|^{2} & \leq\|\phi\|^{2} \int_{-(\delta+1)}^{\delta+1}\left|e^{i k t}\right|^{2} d t \\
& =\frac{\|\phi\|^{2}\left(e^{2|\operatorname{Im} k|(\delta+1)}-e^{-2|\operatorname{Im} k|(\delta+1)}\right)}{2|\operatorname{Im} k|} .
\end{aligned}
$$

Hence

$$
e^{-2|\operatorname{Im} k|(\delta+1)}|E(k)|^{2} \leq \frac{\|\phi\|^{2}\left(1-e^{-4|\operatorname{Im} k|(\delta+1)}\right)}{2|\operatorname{Im} k|},
$$

which implies that $e^{-|\operatorname{Im} k|(\delta+1)}|E(k)|$ goes to zero as $|\operatorname{Im} k|$ tends to infinity. Furthermore, we have

$$
\begin{aligned}
|T(k)| \leq & 2+\frac{B+C}{2}|\cos (k(\delta-1))| \\
& +\frac{|B-C|}{2}|\cos (k(\delta+1))| \\
\leq & 2+\frac{B+C}{2} \frac{e^{-|\operatorname{Im} k|(\delta-1)}+e^{|\operatorname{Im} k|(\delta-1)}}{2} \\
& +\frac{|B-C|}{2} \frac{e^{-|\operatorname{Im} k|(\delta+1)}+e^{|\operatorname{Im} k|(\delta+1)}}{2} .
\end{aligned}
$$

Hence

$$
\begin{aligned}
e^{-|\operatorname{Im} k|(\delta+1)}|T(k)| & \\
\leq & 2 e^{-|\operatorname{Im} k|(\delta+1)} \\
& +\frac{B+C}{4}\left(e^{-2|\operatorname{Im} k| \delta}+e^{-2|\operatorname{Im} k|}\right) \\
& +\frac{|B-C|}{4}\left(1+e^{-2|\operatorname{Im} k|(\delta+1)}\right),
\end{aligned}
$$

which implies that $e^{-|\operatorname{Im} k|(\delta+1)}|T(k)|$ goes to $|B-C| / 4$ as $|\operatorname{Im} k|$ goes to infinity.

Suppose that $k_{j}, j=1,2 \ldots$, are transmission eigenvalues, a sequence of the zeros of $d(k)$ such that $\left|\operatorname{Im} k_{j}\right| \rightarrow \infty$ as $j \rightarrow \infty$. Then from the above discussion, we have $e^{-\left|\operatorname{Im} k_{j}\right|(\delta+1)}\left|E\left(k_{j}\right)\right| \rightarrow 0$ and $e^{-\left|\operatorname{Im} k_{j}\right|(\delta+1)}\left|T\left(k_{j}\right)\right| \rightarrow|B-C| / 4$ as $j \rightarrow \infty$, but $0=d\left(k_{j}\right)=T\left(k_{j}\right)+E\left(k_{j}\right)$; this leads to a contradiction. Hence if complex eigenvalues exist, all of them lie in a strip parallel to the real axis.

\section{The Existence of Complex Transmission Eigenvalues for Constant $n(x)$}

We consider the eigenvalue problem (1) again and make the assumption that the graph of $n(x)$ is symmetric about $x=$ $1 / 2$; that is, $n(x)=n(1-x)$. Using the change of variables

$$
\begin{array}{cc}
y=x-\frac{1}{2}, \quad \varphi(y)=u(x), \\
\psi(y)=v(x), \quad m(y)=n\left(x-\frac{1}{2}\right),
\end{array}
$$

we can transform (1) into

$$
\begin{gathered}
\varphi^{\prime \prime}+k^{2} m(y) \varphi=0, \quad y \in\left[-\frac{1}{2}, \frac{1}{2}\right], \\
\psi^{\prime \prime}+k^{2} \psi=0, \quad y \in\left[-\frac{1}{2}, \frac{1}{2}\right], \\
\varphi\left(-\frac{1}{2}\right)=\psi\left(-\frac{1}{2}\right), \quad \varphi\left(\frac{1}{2}\right)=\psi\left(\frac{1}{2}\right), \\
\varphi^{\prime}\left(-\frac{1}{2}\right)=\psi^{\prime}\left(-\frac{1}{2}\right), \quad \varphi^{\prime}\left(\frac{1}{2}\right)=\psi^{\prime}\left(\frac{1}{2}\right) .
\end{gathered}
$$

In the symmetric domain, we can separate (47) into two problems.

Lemma 8. In the case when $n(x)=n(1-x), k$ is a transmission eigenvalue if and only if $k$ satisfies $d_{1}(k)=0$ or $d_{2}(k)=0$, where

$$
\begin{aligned}
& d_{1}(k):=k \varphi_{1}\left(\frac{1}{2}\right) \sin \left(\frac{k}{2}\right)+\varphi_{1}^{\prime}\left(\frac{1}{2}\right) \cos \left(\frac{k}{2}\right), \\
& d_{2}(k):=k \varphi_{2}\left(\frac{1}{2}\right) \cos \left(\frac{k}{2}\right)-\varphi_{2}^{\prime}\left(\frac{1}{2}\right) \sin \left(\frac{k}{2}\right) .
\end{aligned}
$$

$\varphi_{1}$ and $\varphi_{2}$ are defined in (49). 
Proof. By the fact that $m(-y)=m(y)$ for $y \in[-1 / 2,1 / 2]$, we know $\varphi(-y)$ satisfies the first equation in $(47)$ if $\varphi(y)$ is a solution to that equation. Hence there exist an even function $\varphi_{1}(y)$ and an odd function $\varphi_{2}(y)$ such that

$$
\begin{array}{rlrl}
\varphi_{i}^{\prime \prime}+k^{2} m(y) \varphi_{i} & =0, & y \in\left[-\frac{1}{2}, \frac{1}{2}\right], i=1,2, \\
\varphi_{1}(0) & =1, & \varphi_{1}^{\prime}(0) & =0, \\
\varphi_{2}(0) & =0, & \varphi_{2}^{\prime}(0) & =1 .
\end{array}
$$

So the solutions $\varphi$ and $\psi$ for (47) can be written as

$$
\begin{gathered}
\varphi(y)=c_{1} \varphi_{1}(y)+c_{2} \varphi_{2}(y), \\
\psi(y)=d_{1} \cos (k y)+d_{2} \sin (k y),
\end{gathered}
$$

where $c_{1}, c_{2}, d_{1}$, and $d_{2}$ are constants. Basing on the properties of even and odd functions, we get that, for all $y \in[-1 / 2,1 / 2]$,

$$
\begin{aligned}
& \varphi_{1}(-y)=\varphi_{1}(y), \quad \varphi_{2}(-y)=-\varphi_{2}(y), \\
& \varphi_{1}^{\prime}(-y)=-\varphi_{1}^{\prime}(y), \quad \varphi_{2}^{\prime}(-y)=\varphi_{2}^{\prime}(y) .
\end{aligned}
$$

From the boundary conditions in (47), we get

$$
\begin{aligned}
& c_{1} \varphi_{1}\left(-\frac{1}{2}\right)+c_{2} \varphi_{2}\left(-\frac{1}{2}\right)=d_{1} \cos \left(\frac{k}{2}\right)-d_{2} \sin \left(\frac{k}{2}\right), \\
& c_{1} \varphi_{1}\left(\frac{1}{2}\right)+c_{2} \varphi_{2}\left(\frac{1}{2}\right)=d_{1} \cos \left(\frac{k}{2}\right)+d_{2} \sin \left(\frac{k}{2}\right), \\
& c_{1} \varphi_{1}^{\prime}\left(-\frac{1}{2}\right)+c_{2} \varphi_{2}^{\prime}\left(-\frac{1}{2}\right)=d_{1} k \sin \left(\frac{k}{2}\right)+d_{2} k \cos \left(\frac{k}{2}\right), \\
& c_{1} \varphi_{1}^{\prime}\left(\frac{1}{2}\right)+c_{2} \varphi_{2}^{\prime}\left(\frac{1}{2}\right)=-d_{1} k \sin \left(\frac{k}{2}\right)+d_{2} k \cos \left(\frac{k}{2}\right) .
\end{aligned}
$$

From (51), (52) and (53) yield that

$$
\begin{aligned}
& c_{1} \varphi_{1}\left(\frac{1}{2}\right)=d_{1} \cos \left(\frac{k}{2}\right), \\
& c_{2} \varphi_{2}\left(\frac{1}{2}\right)=d_{2} \sin \left(\frac{k}{2}\right) .
\end{aligned}
$$

Using (51), (54), and (55), we obtain that

$$
\begin{aligned}
& -c_{1} \varphi_{1}^{\prime}\left(\frac{1}{2}\right)=d_{1} k \sin \left(\frac{k}{2}\right), \\
& c_{2} \varphi_{2}^{\prime}\left(\frac{1}{2}\right)=d_{2} k \cos \left(\frac{k}{2}\right) .
\end{aligned}
$$

If $c_{1} \neq 0$, then $d_{1} \neq 0$. Equations (56) and (58) imply that

$$
d_{1}(k)=k \varphi_{1}\left(\frac{1}{2}\right) \sin \left(\frac{k}{2}\right)+\varphi_{1}^{\prime}\left(\frac{1}{2}\right) \cos \left(\frac{k}{2}\right)=0 .
$$

In the case when $c_{2} \neq 0$, then $d_{2} \neq 0$. From (57) and (59), we have that

$$
d_{2}(k)=k \varphi_{2}\left(\frac{1}{2}\right) \cos \left(\frac{k}{2}\right)-\varphi_{2}^{\prime}\left(\frac{1}{2}\right) \sin \left(\frac{k}{2}\right)=0 .
$$

If $k$ is a transmission eigenvalue, $c_{1}$ and $c_{2}$ cannot be zero simultaneously. Hence, $d_{1}(k)=0$ or $d_{2}(k)=0$. This completes the proof.

Remark 9. In the case when $n(x)=n(1-x)$, the set of transmission eigenvalues is the union of the sets of $k$-values such that $d_{1}(k)=0$ and $d_{2}(k)=0$.

From now on, we further assume that $m(y)=n(x-1 / 2)$ is a positive constant not equal to 1 on $[0,1]$, by using $n$ to denote that constant value. In this case,

$$
\varphi_{1}(y)=\cos (k \sqrt{n} y), \quad \varphi_{2}(y)=\frac{\sin (k \sqrt{n} y)}{k \sqrt{n}} .
$$

Substituting $\varphi_{1}$ and $\varphi_{2}$ into (48), we have that

$$
\begin{gathered}
d_{1}(k):=k \cos \left(\frac{k \sqrt{n}}{2}\right) \sin \left(\frac{k}{2}\right) \\
-k \sqrt{n} \sin \left(\frac{k \sqrt{n}}{2}\right) \cos \left(\frac{k}{2}\right), \\
d_{2}(k):=\frac{1}{\sqrt{n}} \sin \left(\frac{k \sqrt{n}}{2}\right) \cos \left(\frac{k}{2}\right)-\cos \left(\frac{k \sqrt{n}}{2}\right) \sin \left(\frac{k}{2}\right) .
\end{gathered}
$$

Our goal is to determine under what conditions there exist complex eigenvalues $k$ when $n \neq 1$ is a constant. In this case, $B=\sqrt{n}+1 / \sqrt{n}, C=2, \delta=\sqrt{n}$, and $B>C$. From (28) and (33), we know that there is an infinite number of complex transmission eigenvalues when $0<\sqrt{n}<1$ and $1<$ $\sqrt{n}<3$. A question naturally arises: are there some complex transmission eigenvalues when $\sqrt{n} \geq 3$ ? The following results (see [3]) play central roles in what follows. We give them as lemmas.

Lemma 10 (Laguerre). Let $f(z)$ be a real entire-valued function of order less than 2 with all its zeros being real. Then the critical points of $f(z)$ (i.e., the zeros of $f^{\prime}(z)$ ) are also real and interlace those of $f(z)$.

Lemma 11. Let $f(z)$ be a real-valued entire function of order less than 2. Then if all its zeros are real, it cannot have more than one critical point inside an interval where it does not change sign.

Lemma 12. Let $f(z)$ be a real-valued entire function of order less than 2. Suppose that $f(z)$ has infinitely many real zeros and only a finite number of complex ones. Then $f(z)$ has a single critical point on each interval $\left(a_{l}, a_{l+1}\right)$ formed by two consecutive real zeros of $f(z)$ when the interval is sufficiently far away from the origin.

In order to find the transmission eigenvalues, we study the roots of $d_{1}=0$ and $d_{2}=0$. First of all, we give some illustrative examples. 
Example 13. First, when $\sqrt{n}=2$, we have that

$$
\begin{gathered}
d_{1}(k)=-k \sin \left(\frac{k}{2}\right)(2+\cos (k)), \\
d_{2}(k)=\sin \left(\frac{k}{2}\right)(1-\cos (k)) ;
\end{gathered}
$$

hence $d(k)$ has a simple zero at $k=0$, an infinite set of real zeros of multiplicity 3 at $k$-values $2 j \pi$ for $j \in \mathbb{N}$, and an infinite set of simple complex zeros at $k$-values that are given by

$$
(2 j-1) \pi+i \log (2 \pm \sqrt{3}), \quad j \in \mathbb{N} \text {. }
$$

Second, when $\sqrt{n}=3$, we get

$$
\begin{gathered}
d_{1}(k)=-2 k \sin k(1+\cos k), \\
d_{2}(k)=2 \sin k(-1+\cos k) ;
\end{gathered}
$$

hence $d(k)$ has a simple zero at $k=0$ and an infinite set of real zeros of multiplicity 3 at $k$-values $j \pi$ for $j \in \mathbb{N}$. Third, when $\sqrt{n}=2 / 3$, then

$$
\begin{gathered}
d_{1}(k)=\frac{k}{3} \sin \left(\frac{k}{6}\right)\left(2 \cos ^{2}\left(\frac{k}{3}\right)+\cos \left(\frac{k}{3}\right)+2\right), \\
d_{2}(k)=-\sin ^{3}\left(\frac{k}{6}\right)\left(3+2 \cos \left(\frac{k}{3}\right)\right) .
\end{gathered}
$$

So $d(k)$ has a simple zero at $k=0$, an infinite set of real zeros of multiplicity 4 at $k$-values $6 j \pi$ for $j \in \mathbb{N}$, an infinite set of simple complex zeros at $k$-values

$$
3(2 j+1) \pi+i 3 \log \left(\frac{3 \pm \sqrt{5}}{2}\right), \quad j \in \mathbb{N}
$$

and an infinite set of complex zeros at $k$-values such that

$$
2 \cos ^{2}\left(\frac{k}{3}\right)+\cos \left(\frac{k}{3}\right)+2=0
$$

As we can see from the above examples, this problem may only have real transmission eigenvalues under some conditions. The following theorem presents a sufficient and necessary condition for the nonexistence of complex transmission eigenvalues in the case when $n$ is constant.

Theorem 14. Let $n$ be a constant not equal to 1 . Then all of the transmission eigenvalues are real when $\sqrt{n}$ is an odd number or a reciprocal of an odd number. Otherwise, (1) has infinitely many real and complex transmission eigenvalues.
Proof. We see that if $0<n<1$, then $\eta:=1 / n>1$. Set $\lambda=\sqrt{n} k$, and we have

$$
\begin{aligned}
d_{1}(k)= & \lambda\left(\sqrt{\eta} \cos \left(\frac{\lambda}{2}\right) \sin \left(\frac{\sqrt{\eta} \lambda}{2}\right)\right. \\
& \left.-\sin \left(\frac{\lambda}{2}\right) \cos \left(\frac{\sqrt{\eta} \lambda}{2}\right)\right), \\
d_{2}(k)= & \sqrt{\eta} \sin \left(\frac{\lambda}{2}\right) \cos \left(\frac{\sqrt{\eta} \lambda}{2}\right) \\
& -\cos \left(\frac{\lambda}{2}\right) \sin \left(\frac{\sqrt{\eta} \lambda}{2}\right) .
\end{aligned}
$$

So it suffices to consider the case $n>1$ only. The zeros of $d_{11}(k):=d_{1}(k) / k$ and $d_{2}(k)$ are the critical points of the functions

$$
f_{1}(k):=\frac{\cos (k \sqrt{n} / 2)}{\cos (k / 2)}, \quad f_{2}(k):=\frac{\sin (k \sqrt{n} / 2)}{\sin (k / 2)},
$$

separately. Obviously, $f_{1}(k)$ is a real-valued entire function of order less than 2 with all its zeros being real when $\sqrt{n}$ is an odd number, and if $\sqrt{n}$ is an integer, $f_{2}(k)$ is a real-valued entire function of order less than 2 with all its zeros being real. It follows from Laguerre's theorem that all roots of $d_{11}(k)=0$ are real when $\sqrt{n}$ is an odd number, and the roots of $d_{2}(k)=0$ are real when $\sqrt{n}$ is an integer. Hence, from Lemma 8, (47) only has real transmission eigenvalues when $\sqrt{n}$ is an odd number.

For the second part of this theorem, we only need to show that $d_{11}(k)$ has an infinite number of complex roots when $\sqrt{n}$ is not an odd number. We note that

$$
d_{11}^{\prime}(k)=\frac{1-n}{2} \cos \frac{k \sqrt{n}}{2} \cos \frac{k}{2}
$$

which has zeros at

$$
\begin{gathered}
\{\pi, 3 \pi, \ldots,(2 N+1) \pi, \ldots\}, \\
\left\{\frac{\pi}{\sqrt{n}}, \frac{3 \pi}{\sqrt{n}}, \ldots, \frac{(2 j+1) \pi}{\sqrt{n}}, \ldots\right\}, \quad N, j \in \mathbb{N} .
\end{gathered}
$$

According to Lemmas 11 and 12, our aim here is to argue that there are infinitely many intervals where $d_{11}(k)$ does not change sign and has at least two consecutive critical points inside.

From this point on, take $N>0$ to be a fixed integer. If $\sqrt{n}(N+1 / 2)-1 / 2 \notin \mathbb{Z}$, there is an integer $j$, such that $\sqrt{n}(N+$ $1 / 2)-1 / 2<j<\sqrt{n}(N+1 / 2)+1 / 2$; that is,

$$
\sqrt{n}\left(N+\frac{1}{2}\right) \pi<\left(j+\frac{1}{2}\right) \pi<\sqrt{n}\left(N+\frac{1}{2}\right) \pi+\pi .
$$

Then we have

$$
\left(j+\frac{1}{2}\right) \pi=\sqrt{n}\left(N+\frac{1}{2}\right) \pi+\epsilon \pi \quad \text { for some } \epsilon \in(0,1) .
$$


From (74), we have

$$
(2 N+1) \pi-\frac{(2 j+1) \pi}{\sqrt{n}}<\frac{2 \pi}{\sqrt{n}} .
$$

So $(2 N+1) \pi$ and $(2 j+1) \pi / \sqrt{n}$ are two consecutive critical points of $d_{11}(k)$. At $(2 N+1) \pi$,

$$
\begin{aligned}
d_{11}((2 N+1) \pi) & =(-1)^{N} \cos \left(\sqrt{n}\left(N+\frac{1}{2}\right) \pi\right) \\
& =(-1)^{N} \cos \left(\left(j+\frac{1}{2}\right) \pi-\epsilon \pi\right) \\
& =(-1)^{N+1} \sin (j \pi-\epsilon \pi) \\
& =(-1)^{N+j} \sin (\epsilon \pi) .
\end{aligned}
$$

At the critical point $(2 j+1) \pi / \sqrt{n}$,

$$
\begin{aligned}
d_{11}\left(\frac{(2 j+1) \pi}{\sqrt{n}}\right) & =(-1)^{j+1} \sqrt{n} \cos \left(\frac{(j+1 / 2) \pi}{\sqrt{n}}\right) \\
& =(-1)^{j+1} \sqrt{n} \cos \left(\left(N+\frac{1}{2}\right) \pi+\frac{\epsilon \pi}{\sqrt{n}}\right) \\
& =(-1)^{j} \sqrt{n} \sin \left(N \pi+\frac{\epsilon \pi}{\sqrt{n}}\right) \\
& =(-1)^{j+N} \sqrt{n} \sin \left(\frac{\epsilon \pi}{\sqrt{n}}\right) .
\end{aligned}
$$

We see that the signs of $d_{11}((2 N+1) \pi)$ and $d_{11}((2 j+1) \pi / \sqrt{n})$ are identical since $\epsilon \in(0,1)$ and $\sqrt{n}>1$; that is, $d_{11}(k)$ cannot change sign in $((2 j+1) \pi / \sqrt{n},(2 N+1) \pi)$.

In the case when $\sqrt{n}(N+1 / 2)-1 / 2 \in \mathbb{Z}$, there is an integer $j$ such that

$$
j<\sqrt{n}\left(N+\frac{1}{2}\right)-\frac{1}{2}<j+1 .
$$

We have

$$
\left(j+\frac{1}{2}\right) \pi<\sqrt{n}\left(N+\frac{1}{2}\right) \pi<\left(j+\frac{1}{2}\right) \pi+\pi .
$$

Then

$$
\sqrt{n}\left(N+\frac{1}{2}\right) \pi=\left(j+\frac{1}{2}\right) \pi+\epsilon \pi,
$$

for some $\epsilon \in(0,1)$. In the same way as done above, we have that $d_{11}(k)$ cannot change sign in $((2 N+1) \pi,(2 j+1) \pi / \sqrt{n})$.

\section{Conflict of Interests}

The authors declare that there is no conflict of interests regarding the publication of this paper.

\section{Acknowledgment}

This research was supported by the National Natural Science Foundation of China under Grant no. 61170019.

\section{References}

[1] D. Colton and R. Kress, Inverse Acoustic and Electromagnetic Scattering Theory, Springer, Berlin, Germany, 3rd edition, 2012.

[2] F. Cakoni, D. Colton, and D. Gintides, "The interior transmission eigenvalue problem," SIAM Journal on Mathematical Analysis, vol. 42, no. 6, pp. 2912-2921, 2010.

[3] Y. Leung and D. Colton, "Complex transmission eigenvalues for spherically stratified media," Inverse Problems, vol. 28, no. 7, Article ID 075005, 2012.

[4] D. Colton and Y. Leung, "Complex eigenvalues and the inverse spectral problem for transmission eigenvalues," Inverse Problems, vol. 29, no. 10, Article ID 104008, 2013.

[5] J. Sylvester, "Transmission eigenvalues in one dimension," Inverse Problems, vol. 29, no. 10, 11 pages, 2013.

[6] J. Poschel and E. Trubowitz, Inverse Spectral Theory, Academic Press, Boston, Mass, USA, 1987.

[7] D. Colton, L. Päivärinta, and J. Sylvester, "The interior transmission problem," Inverse Problems and Imaging, vol. 1, no. 1, pp. 13-28, 2007.

[8] R. M. Young, An Introduction to Nonharmonic Fourier Series, Academic Press, San Diego, Calif, USA, 2001. 


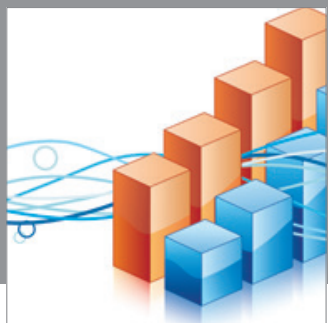

Advances in

Operations Research

mansans

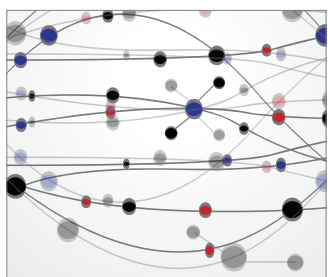

The Scientific World Journal
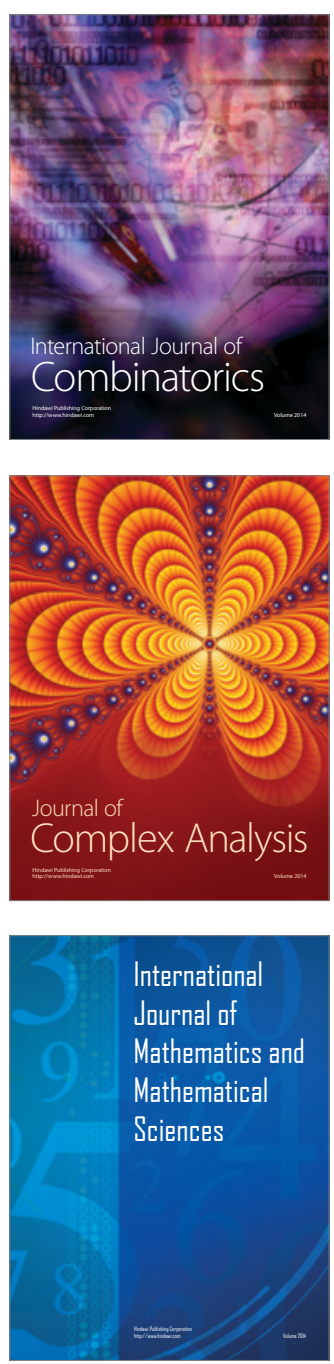
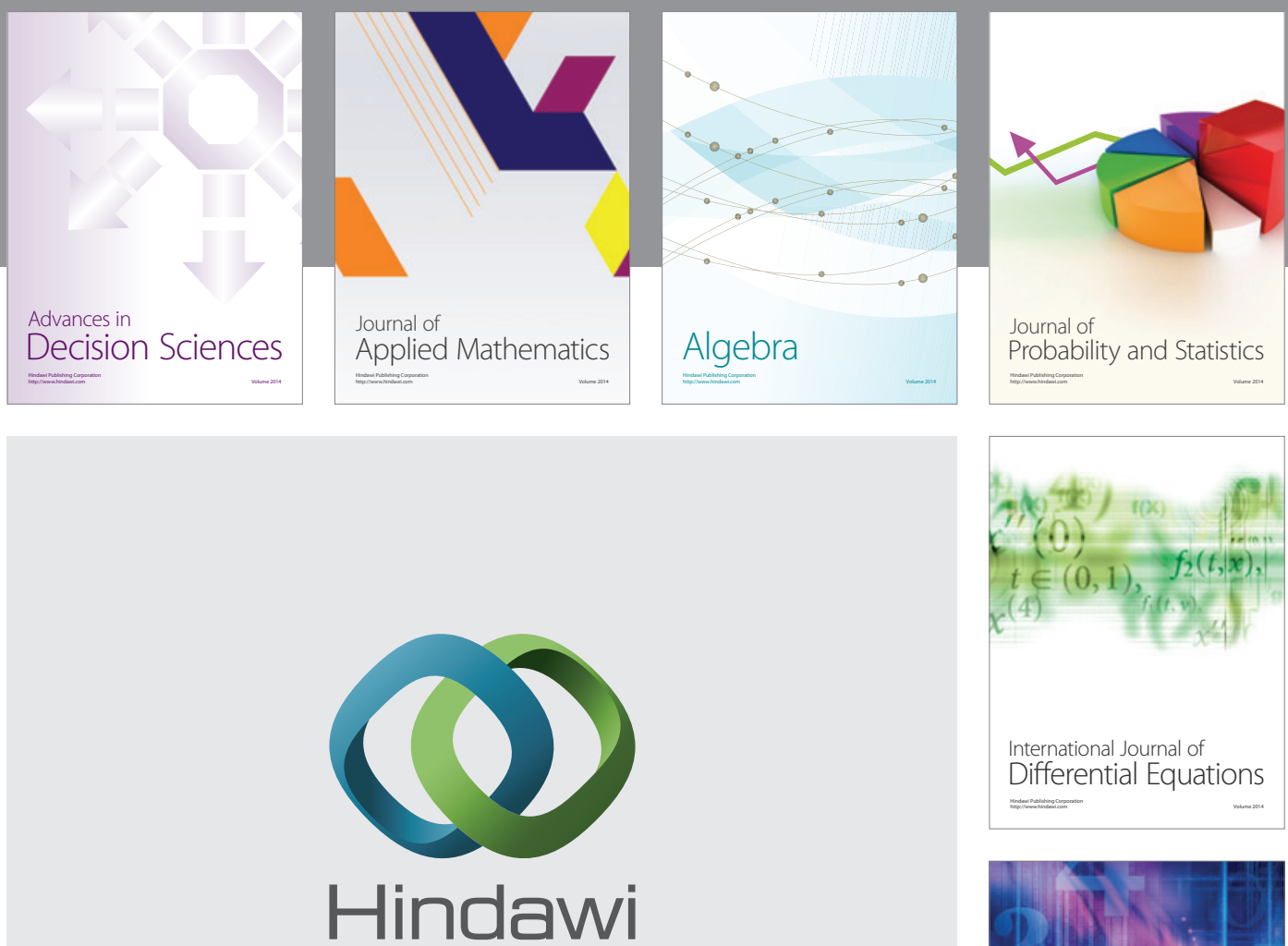

Submit your manuscripts at http://www.hindawi.com
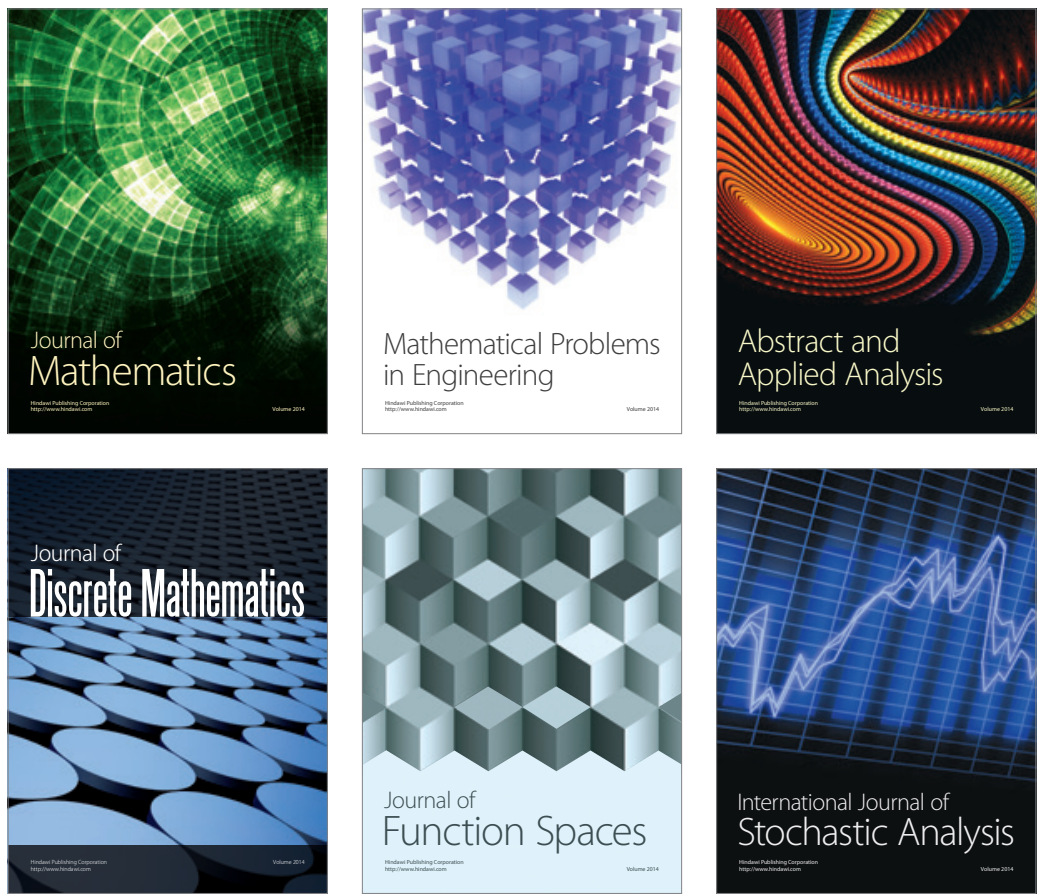

Journal of

Function Spaces

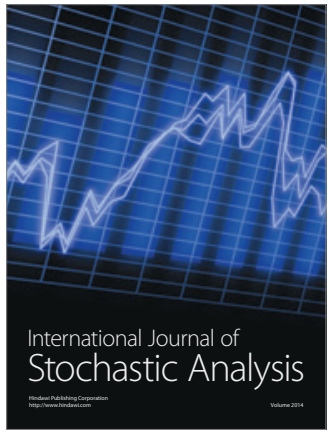

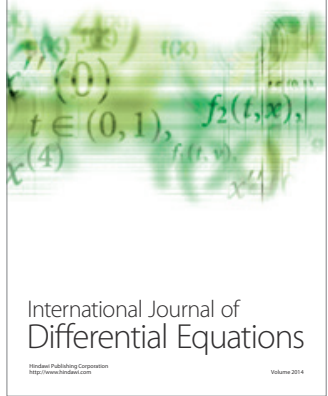
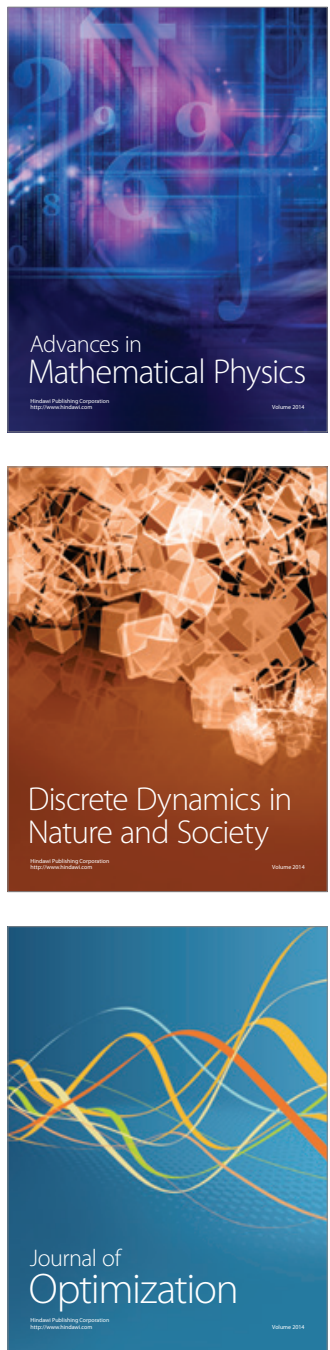